\title{
Bladder cancer in the elderly patient: challenges and solutions
}

This article was published in the following Dove Press journal:

Clinical Interventions in Aging

10 June 2015

Number of times this article has been viewed

\author{
Elizabeth A Guancial' \\ Breton Roussel ${ }^{2}$ \\ Derek P Bergsma ${ }^{3}$ \\ Kevin C Bylund ${ }^{3}$ \\ Deepak Sahasrabudhe \\ Edward Messing ${ }^{4}$ \\ Supriya G Mohile' \\ Chunkit Fung' \\ 'Division of Hematology/Oncology, \\ Department of Medicine, Wilmot \\ Cancer Institute, University of \\ Rochester Medical Center School of \\ Medicine and Dentistry, Rochester, \\ NY, ${ }^{2}$ Robert Wood Johnson Medical \\ School, Rutgers University, New \\ Brunswick, NJ, ${ }^{3}$ Department of \\ Radiation Oncology, Wilmot Cancer \\ Institute, University of Rochester, \\ Rochester, NY, ${ }^{4}$ Department of \\ Urology, University of Rochester, \\ Rochester, NY, USA
}

Correspondence: Elizabeth Guancial Division of Hematology/Oncology, Department of Medicine, Wilmot Cancer Institute, University of Rochester Medical Center School of Medicine and Dentistry, 60I Elmwood Avenue, Box 704, Rochester, NY 14642, USA $\mathrm{Tel}+\mathrm{I} 5852755823$

Fax + I 585273 I05 I

Email elizabeth_guancial@urmc. rochester.edu

\begin{abstract}
Bladder cancer (BC) is an age-associated malignancy with increased prevalence in the elderly population. Elderly patients are a vulnerable population at increased risk for treatmentrelated toxicity secondary to medical comorbidities and geriatric syndromes. As a result, this population has been historically undertreated and suffers worse disease-specific outcomes than younger patients with BC. Recognition of this disparity has led to efforts to individualize treatment decisions based on functional status rather than chronologic age in an effort to optimize the use of curative therapies for the fit elderly and modify treatments to reduce the risk of toxicity and disease-related morbidity in vulnerable or frail patients. The comprehensive geriatric assessment is a decision framework that helps to balance underlying health considerations and risks of therapy with aggressiveness of the cancer. Development of systemic therapies with increased efficacy against BC and reduced toxicity are eagerly awaited, as are techniques and interventions to reduce the morbidity from surgery and radiation for patients with BC.
\end{abstract}

Keywords: bladder cancer, elderly, quality of life, surgery, radiation therapy, chemotherapy

\section{Introduction}

Bladder cancer $(\mathrm{BC})$ is an age-associated malignancy, with a median age at diagnosis of 73 years, and individuals aged 75-84 years accounting for the largest percentage at $30 \%$ of new cases. ${ }^{1}$ Curative therapy with either radical cystectomy (RC), with or without perioperative chemotherapy, or combined-modality therapy (CMT) with a goal of bladder preservation through a combination of maximal transurethral resection of bladder tumor, radiation therapy (RT), and concurrent chemotherapy, offers varying rates of long-term survival dependent upon the stage at diagnosis. The risk of recurrence estimated by a post-RC nomogram ranges from $20 \%$ for patients with organ-confined disease to $70 \%$ for those with limited lymph node involvement. ${ }^{2}$ In contrast, metastatic disease remains incurable with currently available therapies. The average life expectancy for metastatic BC is 14 months in patients who receive systemic treatment and 8 months without treatment, making it the eighth leading cause of cancer-related death in 2013..$^{3,4}$

While treatment options for organ-confined, locally advanced, or metastatic disease are not directly impacted by chronologic age, age-related declines in performance status and medical comorbidities significantly affect the risk of treatment-related toxicity and must be factored into treatment-related decisions in order to optimally deliver patientcentered care. This review summarizes the relevant literature regarding management of muscle-invasive bladder cancer (MIBC) and metastatic BC in light of characteristics unique to the elderly population; describes what is known about health-related quality of life (HRQoL) for BC patients; and discusses clinically available tools to help 
guide management decisions in the elderly, specifically the comprehensive geriatric assessment (GA).

\section{Management of muscle invasive bladder cancer in the elderly Radical cystectomy}

When $\mathrm{BC}$ is not detected until it invades the muscularis propria (at least T2 disease), endoscopic management with transurethral resection of bladder tumor is rarely capable of effecting a cure. RC remains the gold standard treatment for non-metastatic MIBC. This surgery is associated with a high incidence of postoperative morbidity and mortality. In a mixed-aged population undergoing $\mathrm{RC}$, the perioperative mortality rate was $3 \%-7 \%$ and the 90 -day complication rate was $60 \% .{ }^{5}$ The nature of this surgery as a high-risk intervention requires careful selection for surgical candidacy amongst all patients and especially in the elderly population.

Several studies have reported that older patients are more susceptible to complications and poor outcomes from RC. ${ }^{6}$ Schiffmann et al reported in a retrospective study of 5,207 patients that 90-day mortality rates following RC were $6.4 \%, 10.1 \%$, and $14.8 \%$ in patients aged $65-69$ years, 70-79 years, and $\geq 80$ years, respectively. ${ }^{7}$ Additional variables within the elderly population also shown to be associated with worse 90-day mortality include low socioeconomic status, unmarried status, and a greater number of comorbidities. ${ }^{7,8}$ Moderate and severe comorbidities are associated with major early postoperative complications (odds ratio [OR] 11.4, $P<0.001$, and OR 15.2, $P<0.001$ respectively), including return to the operating room, acute coronary syndrome, pulmonary embolism, sepsis, and acute respiratory failure. ${ }^{8}$ Another study reports that advanced age is associated with shorter time to recurrence and worse cancer-specific survival in patients undergoing RC. ${ }^{9}$

Much of the perioperative morbidity associated with RC is due to the complications of urinary diversion. Patients older than 70 years are more likely to receive incontinent forms of urinary diversion, such as an ileal conduit, than continent forms of diversion ( $70 \%$ versus $30 \%$ of cases, respectively). ${ }^{10,11}$ Of those who receive orthotopic bladder substitution, incontinence is reported more often with advanced age, which may be one reason why the elderly are more likely to receive an ileal conduit than younger patients. ${ }^{10}$ However, Sogni et al reported similar quality of life in elderly patients regardless of type of urinary diversion. ${ }^{12}$ In addition, several studies have demonstrated no difference between ileal conduit and orthotopic diversion with respect to early diversion-related complications, late complications, or operative mortality. ${ }^{12,13}$
Well-selected octogenarians may be reasonable surgical candidates for both $\mathrm{RC}$ and continent forms of diversion, and selection should be based on physiologic reserve rather than chronologic age. In a single-institution, retrospective study of 1,054 patients, Clark et al reported similar rates of operative mortality, early diversion-related complications, late complications, and operative mortality among patients aged $<60$ years, $60-69$ years, $70-79$ years, and $>80$ years. ${ }^{13}$ They noted longer postoperative length of hospitalization (on average 1 day) in elderly patients, which is consistent with other reports. ${ }^{13}$ These studies presumably involved relatively healthy elderly adults based on the fact that they were offered RC, although American Society of Anesthesiologists and Easton Cooperative Oncology Group performance scores were not reported in these series.

There is a dearth of robust data comparing laparoscopic versus open RC in older patients. A 2014 meta-analysis looking at 16 small prospective and retrospective studies reported decreased intraoperative blood loss, less narcotic analgesic requirement, and longer operative time in laparoscopic versus open RC. ${ }^{14}$ There appear to be no significant differences in oncologic outcomes when comparing the two modalities, and none of the studies report age-based differences in any outcomes. ${ }^{15}$

Robot-assisted RC is gaining interest as an alternative to open surgery for MIBC but is relatively unstudied in the older patient population. Despite this, the only randomized study evaluating robot-assisted versus open $\mathrm{RC}(\mathrm{n}=118$; average age of patients, 67.4 versus 69.2 years, respectively) reported decreased estimated blood loss $(P<0.001)$, less inhouse analgesia requirement $(P<0.001)$, and longer operative times $(P<0.019)$ in the robot-assisted group, but similar perioperative complication rates and lengths of stay. ${ }^{16}$ Several retrospective studies suggest that there may be benefits to a robot-assisted approach, such as decreased transfusion rates, but similar 90-day postoperative complication rates and oncologic outcomes with either robot-assisted or open RC. ${ }^{5,17}$ A recent study of robot-assisted $\mathrm{RC}$ in octogenarians (median age 83 [80-94] years, 68\% $\geq \mathrm{pT} 2$ ) reported 2-year recurrence free, cancer-specific, and overall survival rates of $73 \%, 74 \%$, and $61 \%$, respectively, which compares favorably with results in younger patients. ${ }^{18}$ However, randomized prospective data examining benefits of robot-assisted versus open RC in the elderly remain limited.

\section{Definitive chemoradiation}

Due to the relatively large proportion of elderly among newly diagnosed patients with MIBC, RC with neoadjuvant or 
adjuvant chemotherapy may not be an appropriate option due to poor physiologic reserve, poor performance status, and/or presence of comorbidities. Current data from the American Cancer Society and the National Cancer Database show that potentially curative treatment is underutilized with only $50 \%-55 \%$ of individuals $>70$ years of age receiving $\mathrm{RC}$, definitive RT, or CMT. ${ }^{19,20} \mathrm{RT}$ for $\mathrm{BC}$ is well-tolerated in the elderly and can provide excellent overall and disease-specific survival when delivered alone or as CMT with chemotherapy. Importantly, short-course palliative RT is an effective tool for relieving symptoms due to $\mathrm{BC}$.

For elderly patients with MIBC who are poor surgical candidates and/or would like to avoid major surgery, selective bladder preservation with CMT where $\mathrm{RC}$ is reserved for initial treatment failure is an excellent option and provides an opportunity for long-term survival with an intact, functioning bladder. Candidates for selective bladder preservation have no nodal involvement ( $\geq$ N1), invasion of adjacent organs (T4), disease near ureteral orifices or bladder trigone with hydronephrosis, or carcinoma in situ; have undergone a maximal, and preferably visibly complete, transurethral resection of bladder tumor; and can tolerate concurrent chemoradiation..$^{21}$ The conceptual approach utilizes approximately 5 weeks of CMT (to approximately 40-45 Gy), typically with either cisplatin or 5-fluorouracil/mitomycin $\mathrm{C}$, followed by repeat cystoscopy to assess response with either salvage cystectomy for patients with residual invasive disease, or completion of CMT to approximately $60-65$ Gy for those with a complete response (CR). It is important for these patients to understand and comply with the need for lifelong surveillance cystoscopy, with prompt salvage cystectomy if local recurrence is detected.

James et al published the results of BC2001, a Phase III randomized controlled trial conducted in the UK that enrolled patients (median age 72 years) with MIBC to RT alone versus RT with a combination regimen of concurrent 5 -fluorouracil and mitomycin C. ${ }^{22}$ The RT dose ranged between 55 and 64 Gy delivered over 4-6.5 weeks. This study demonstrated significantly improved locoregional control of BC with CMT compared with RT alone (5-year locoregional control rates $67 \%$ versus $54 \%$, respectively), and a trend toward improved overall survival with CMT compared with RT alone (5-year overall survival of $48 \%$ versus $35 \%$, respectively; $P=0.16$ ). ${ }^{22}$ Clayman et al examined 93 elderly patients (defined as older than 75 years) enrolled in prospective trials at Massachusetts General Hospital who were treated with selective bladder preservation, with approximately $33 \%$ achieving a CR and nearly $60 \%$ of these patients going on to complete a full course of consolidation CMT. ${ }^{19}$ Disease-specific survival was $60 \%$ at 5 years and $56 \%$ at 10 years, which compares favorably with RC. Mak et al recently published a pooled analysis of long-term outcomes from six Radiation Therapy Oncology Group protocols of selective, bladder-preserving CMT which demonstrated a CR of $69 \%{ }^{23}$ Five-year and 10 -year overall survival rates were $57 \%$ and $36 \%$, respectively, with increased 5-year and 10-year disease-specific survival rates of $71 \%$ and $65 \%$, respectively, similar to outcomes from RC. The 10-year estimates of non-muscle invasive and muscleinvasive local failures were $36 \%$ and $14 \%$, respectively. Elderly patients ( $\geq 75$ years) were less likely to complete CMT compared with younger patients ( $78 \%$ versus $88 \%$, $P=0.28)$. Disease-specific outcomes for elderly patients were comparable with those for younger patients with respect to rates of $\mathrm{CR}$ ( $72 \%$ versus $73 \% ; P=0.78$ ), disease-specific survival ( $71 \%$ versus $70 \% ; P=0.74)$, and bladder preservation at 5 years ( $76 \%$ versus $81 \% ; P=0.55$ ).

Reports from studies of CMT demonstrate low rates of acute and late toxicities. In the BC2001 study, approximately one-third of patients in the CMT arm had grade 3/4 acute toxicities and less than $10 \%$ of patients had grade $3 / 4$ late toxicities at a median follow-up of 69.9 months. ${ }^{22}$ Efstathiou et al corroborated these low rates of significant late pelvic toxicity in a separate publication compiling patients treated in several prospective Radiation Therapy Oncology Group trials reporting $5.7 \%$ grade $3+$ genitourinary and $1.9 \%$ grade $3+$ gastrointestinal late pelvic toxicities at median follow-up of 5.4 years, with no significant difference between patients older than or younger than 65 years. ${ }^{24}$ Several groups have published favorable outcomes in regard to long-term bladder function after chemoradiation. Zietman et al published quality of life and urodynamic results for 71 patients retaining their bladder after chemoradiation, with $75 \%$ of patients having normal bladder function on urodynamic studies and $85 \%$ reporting no or only occasional urgency at 6.3 years median follow-up..$^{25}$

Beyond definitive therapy, RT alone can also be used to palliate local tumor-related symptoms of hematuria, dysuria, or frequency, often with just a few fractions. The Medical Research Council BA-09 trial reported that $68 \%$ of patients with T2-T4a tumors achieved symptomatic improvement, with no difference between three-fraction (in 1 week) and ten-fraction (in 2 weeks) treatment schedules. ${ }^{26}$ Researchers from Greece prospectively studied 58 patients over the age of 75 years with symptomatic $\mathrm{T} 1-\mathrm{T} 2 \mathrm{BC}$, and reported $95 \%$ palliation of hematuria and $67 \%$ improvement in dysuria or frequency with the use of six treatments delivered weekly, with no grade 3 toxicity observed. ${ }^{27}$ 


\section{Chemotherapy: neoadjuvant and adjuvant}

Cisplatin-based neoadjuvant chemotherapy (NAC) improves overall survival for patients with MIBC by approximately $5 \%$ at 5 years compared with RC alone. ${ }^{28}$ Furthermore, NAC doubles the rate of pathologic $\mathrm{CR}$ at the time of RC from $10 \%-15 \%$ to $30 \%$; for patients who experience pathologic CR, the 5-year overall survival is improved to $85 \%{ }^{29}$ Trials of cisplatin-based adjuvant chemotherapy, including a recently published study, have been underpowered to detect a statistically significant improvement in outcomes compared with those having $\mathrm{RC}$ alone largely due to a failure to accrue. ${ }^{30}$ Thus, based on the proven benefit and level of evidence, $\mathrm{NAC}$ is the preferred approach to management for patients with MIBC who are eligible to receive cisplatin-based chemotherapy. ${ }^{31}$

Despite the endorsement of national guidelines, the use of cisplatin-based NAC is limited in contemporary clinical practice, both in the community and at academic centers. ${ }^{32}$ Factors that impact the use of NAC are complex and include misperceptions on the part of both physicians and patients regarding a lack of benefit and increased toxicity. A coordinated multidisciplinary approach to management has been shown to increase use of NAC and is proposed as a quality indicator of care for MIBC. ${ }^{33,34}$ The incorporation of a geriatric oncologist or geriatrician skilled in the administration and interpretation of the comprehensive GA may help to prospectively identify patients at increased risk for chemotherapy-induced toxicity. ${ }^{35}$

While $\mathrm{BC}$ is primarily a disease of the elderly, patients over the age of 65 years are underrepresented in clinical trials for $\mathrm{BC} .{ }^{36} \mathrm{~A}$ meta-analysis of eleven randomized trials of NAC plus RC versus RC alone for MIBC revealed that $42 \%$ of patients who received NAC and $46 \%$ of patients treated with $\mathrm{RC}$ alone were older than 64 years. ${ }^{28}$ As recently discussed by Galsky, variability in the reporting of age of participants in clinical trials hampers efforts to fully understand the experience of elderly patients treated in a research clinical trial. ${ }^{37}$

Cisplatin is the most active cytotoxic agent against BC. However, carboplatin is frequently substituted for cisplatin as first-line therapy for patients with advanced MIBC out of concern for increased toxicity in the elderly. ${ }^{38}$ In the neoadjuvant setting, carboplatin is not an acceptable substitute for cisplatin, given the reduced response rate..$^{38-40}$ An expert panel recently developed a consensus definition for cisplatin ineligibility for the purposes of clinical trial development, although these criteria are applicable to clinical practice as well (Table 1). ${ }^{41}$ This definition provides a reproducible standard for determining which patients are unfit for cisplatin.
Table I Relative exclusion criteria for treatment with cisplatin in patients with metastatic bladder cancer

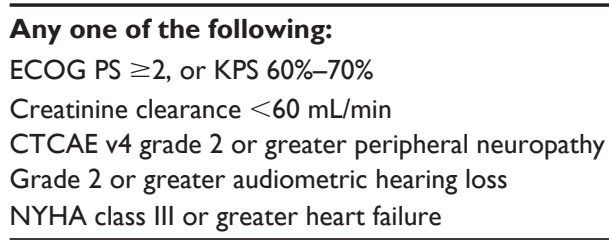

Abbreviations: CTCAE, Common Terminology Criteria for Adverse Events; ECOG PS, Eastern Cooperative Oncology Group Performance Status; KPS, Karnofsky Performance Status; NYHA, New York Heart Association.

For those who are able to undergo treatment with cisplatin, age alone does not appear to affect tolerability or disease outcomes based on available data. A single-institution case series demonstrated similar rates of eligibility for definitive local therapy following cisplatin-based NAC. Additionally, it reported similar clinical outcomes including pathologic $\mathrm{CR}$, overall survival, and relapse-free survival at 3 years in patients aged $\geq 70$ years versus younger patients with MIBC. ${ }^{42}$ A recent study of tolerability and efficacy of cisplatin-based combination chemotherapy in metastatic disease based on data from eight Phase II and III clinical trials with a total of 543 patients demonstrated no significant differences in the frequency of renal failure (grade 3-4), febrile neutropenia, or treatment-related death or median survival (12.1 months versus 12.8 months; $P=0.91$ ) for patients older and younger than 70 years of age. ${ }^{43}$

Therefore, for elderly patients who are surgical candidates and have no contraindication for cisplatin-based chemotherapy, NAC is an appropriate management strategy that should be discussed. Patients who are ineligible for cisplatin but acceptable surgical candidates should consider RC alone without chemotherapy. For elderly patients who are medically inoperable due to surgical risk or choose not to undergo $\mathrm{RC}$ but have organ-confined disease and could tolerate cisplatin, CMT can be offered with a cisplatin-based regimen. Alternatively, for those who are ineligible for cisplatin, a regimen of mitomycin $\mathrm{C}$ and 5-fluorouracil can be used.

\section{Management of metastatic bladder cancer in the elderly}

Cisplatin-based combination chemotherapy is recommended as first-line systemic therapy for patients with metastatic BC based on a relative risk of nearly $50 \%{ }^{44}$ The combination of methotrexate, vinblastine, adriamycin, and cisplatin (MVAC) was compared with the doublet of gemcitabine and cisplatin (GC) in a randomized Phase III study of patients with advanced or metastatic BC, and was shown to have comparable outcomes 
(median overall survival 15.2 versus 14 months, respectively) with increased rates of febrile neutropenia (14\% versus $2 \%$ ), grade $3 / 4$ mucositis ( $22 \%$ versus $1 \%$ ), and toxic death $(3 \%$ versus $1 \%$ ) in patients who received MVAC. More patients who received GC reported improved fatigue and performance status while on therapy compared with those treated with MVAC, although differences between treatment arms were not statistically significant. ${ }^{44}$ Therefore, GC is preferred over MVAC as first-line therapy for metastatic disease, and while not supported by randomized data in the neoadjuvant setting, GC is commonly used as NAC as well. ${ }^{31}$

Carboplatin may be substituted for cisplatin for patients unfit to receive cisplatin. Similar to the results of GC versus MVAC in metastatic disease, gemcitabine/carboplatin produced similar overall survival as methotrexate/carboplatin/ vinblastine in cisplatin-ineligible patients with metastatic BC (9.3 months versus 8.1 months, respectively), and was also associated with decreased toxicity. ${ }^{45}$ In a recent Medicare database study, nearly $50 \%$ of patients over the age of 65 years did not receive any chemotherapy for their advanced BC. Among those who did, approximately 50\% received carboplatin, $40 \%$ received cisplatin, and $10 \%$ received a non-platinum containing regimen. ${ }^{46}$

No therapies have been approved by the US Food and Drug Administration (FDA) for second-line treatment following the use of a platinum agent. Some single agents, such as taxanes, pemetrexed, and ifosfamide, were shown to be active against $\mathrm{BC}$ and may be considered in patients eligible for additional therapy. ${ }^{47-50}$ In patients with reduced performance status, single agents may be considered over combination therapy, although the decision to proceed with any systemic therapy rather than best supportive care alone under these conditions must be discussed with the patient. Factors associated with increased chemotherapy-associated toxicity have been prospectively identified in the elderly and include variables that are measured in routine oncology practice and in the GA (Table 2). ${ }^{35}$

Participation in therapeutic clinical trials is strongly encouraged for all eligible patients with $\mathrm{BC}$. There is awareness in the $\mathrm{BC}$ research community of the need for effective and tolerable therapies for elderly patients with $\mathrm{BC}$ and the importance of designing trials that do not exclude this vulnerable population. ${ }^{46}$ While the adverse effects from cytotoxic chemotherapy preclude treatment for many elderly patients, new classes of agents under development may offer a more tolerable side effect profile. Recent advances in the molecular understanding of $\mathrm{BC}$ highlight the genetic heterogeneity of this disease and reveal unique intrinsic subtypes that may
Table 2 Risk factors for chemotherapy toxicity in the elderly

Age $\geq 72$ years

Cancer type (gastrointestinal or genitourinary)

Standard dosing of chemotherapy

Polychemotherapy

Hemoglobin (males $<\mathrm{II} \mathrm{g} / \mathrm{dL}$; females $<10 \mathrm{~g} / \mathrm{dL}$ )

Creatine clearance $<34 \mathrm{~mL} / \mathrm{min}$

Hearing impairment

At least one fall in the last 6 months

Limited in walking one block

Need for assistance in taking medications

Decreased social activities because of physical or emotional health

Note: Data from Montgomery et al..$^{35}$

have treatment implications. ${ }^{51-53}$ Targeted therapies have yet to benefit the majority of patients with $\mathrm{BC}$; however, reports of dramatic and prolonged responses to the mammalian target of rapamycin inhibitor, everolimus, exist in select patients with metastatic BC. ${ }^{54,55}$ The frequent prevalence of predominantly non-overlapping actionable mutations in $\mathrm{BC}$, such as TSC1, BRAF, ERBB2, FGFR1, FGFR3, PIK3CA, PTEN, and $C C N D 1$, has been confirmed by multiple groups. ${ }^{56,57}$ Future trials will select targeted therapies based upon the presence of these potentially predictive mutations.

In addition to targeted therapy, immunotherapy with immune checkpoint blockade of programmed-death ligand 1 (PD-L1) may be a therapeutic option for elderly BC patients given the overall tolerability and lack of significant toxicity of these agents based on the studies published to date. ${ }^{58}$ Promising results in metastatic $\mathrm{BC}$ were recently reported by Powles et al on the activity of MPDL3280A, an anti-PD-L1 antibody, which received breakthrough therapy designation for BC from the FDA in June, 2014, based on these data. ${ }^{59}$ In this adaptive design, Phase I expansion study of MPDL3280A in previously-treated metastatic BC, immunohistochemistry was used to measure expression of PD-L1 in tumor-infiltrating immune cells (macrophages, dendritic cells, and lymphocytes). Objective response rates at 6 weeks was $43 \%$ for patients with immunohistochemistry 2 or 3 tumors, with $7 \% \mathrm{CR}$, and $11 \%$ response rate for immunohistochemistry 0 or 1 tumors. The age of participants in this study ranged from 36 to 86 years with a median of 65 years, and approximately one-third of participants had impaired renal function with creatinine clearance of less than $60 \mathrm{~mL} / \mathrm{min}$. Grade 3 treatment-related adverse events were reported in $4 \%$ of patients; no grade $4 / 5$ events occurred. Therefore, despite advanced age and impairment of renal function, significant toxicity was rare. MK-3475, also known as pembrolizumab, received accelerated FDA approval for treatment of advanced melanoma based on high response 
rates and sustained duration of response in early studies in this disease. ${ }^{60}$ As with MPDL3280A, MK-3475 is associated with less than $5 \%$ grade 3 or higher treatment-related adverse events, although this was in patients with advanced melanoma not BC. ${ }^{61}$ Additional studies of MPDL3280A and anti-PD-1 agents, including MK-3475, are under development for BC. Given the lack of new therapies for this disease in over 30 years, the results from these studies are eagerly awaited.

\section{Health-related quality of life for patients with bladder cancer}

Many older patients with BC have comorbid conditions and impairments of activities of daily living, which clinicians must consider when making therapeutic recommendations, as the competing risk of non-cancer mortality is higher in this population. ${ }^{62-64}$ Several studies have reported that fewer elderly BC patients underwent definitive treatment when compared with their younger counterparts, and this may partially explain the negative effect of age on overall and BC-specific survival. ${ }^{9,63,64}$ To improve BC outcomes, it will not only be imperative to develop standardized decisionmaking models to incorporate HRQoL into the treatment of older BC patients, but to also examine interventions, such as exercise or GA, that can potentially improve HRQoL and in turn the tolerability of various treatments by BC patients. ${ }^{65,66}$ As summarized by the Eighth Annual Bladder Cancer Think Tank sponsored by the Bladder Cancer Advocacy Network, interventions to enhance quality of life for BC patients is an urgent, unmet need and should be a research priority. ${ }^{67}$

Several HRQoL questionnaire instruments are used to assess broad cancer treatment side effects, including the European Organization for Research and Treatment of Cancer Quality of Life Questionnaire version 3.0 QLQ-C30 (EORTC QLQ-C30), and Functional Assessment of Cancer Therapy-General. ${ }^{68-71}$ To measure BC-specific side effects, the EORTC-QLQ-BLM30 and the Functional Assessment of Cancer Therapy-Bladder are commonly utilized. ${ }^{71}$

Data about the long-term burden of BC and its treatment on HRQoL are sparse. ${ }^{72}$ Most of the HRQoL research in BC has focused on the impact of RC and various urinary diversions on HRQoL of patients with MIBC, whereas HRQoL of patients with non-MIBC have rarely been studied. ${ }^{72,73}$ Most studies of HRQoL in BC survivors thus far have been compromised by small samples (ranging from 259 to 525 patients) or poor response rates $(30.8 \%-51.0 \%) .{ }^{65,74,75}$

Recently, two large studies have examined the effects of BC diagnosis on HRQoL using the Surveillance, Epidemiology and End Results-Medicare Health Outcomes
Survey dataset. ${ }^{62,76}$ The first investigation compared prospective changes in HRQoL among 1,432 older patients ( $\geq 65$ years) diagnosed with cancer across nine cancer sites that occur within 2 years of their diagnosis with changes in HRQoL in a similar time frame among a set of matched control subjects without cancers. ${ }^{76}$ The mean physical component score $(\mathrm{PCS})$ of BC patients $(\mathrm{n}=89)$ decreased significantly by 4.3 points $(95 \%$ confidence interval $[\mathrm{CI}]-2.5,-6.1)$ over 2 years, whereas the decrease in the mean mental component score (MCS) was not statistically significant. The second investigation is a cross-sectional study of $1,476 \mathrm{BC}$ patients (aged $\geq 65$ years) that compared HRQoL among those before and after $\mathrm{BC}$ diagnosis. ${ }^{62}$ Fung et al reported significant differences in mean PCS and MCS scores between the pre-BC and post-BC diagnosis cohorts. ${ }^{62}$ Among patients with nonMIBC, the differences in the PCS $(-1.9 ; P<0.01)$ and MCS $(-1.4, P=0.01)$ scores were statistically significant but not in a clinically meaningful way since the differences were less than 5 points. ${ }^{77}$ In contrast, there was a statistically and clinically significant difference in the PCS score $(-5.3 ; P<0.01)$ for patients with MIBC, whereas the difference in the MCS score was not statistically significant $(-2.7, P=0.07)$.

Among patients with MIBC, decreases in HRQoL develop after RC with urinary tract reconstruction (ie, ileal conduit, continent cutaneous diversion, and orthotropic neobladder), particularly in the sexual and urinary domains. ${ }^{68,78}$ Despite the many studies that compared various urinary reconstructive approaches after $\mathrm{RC}$, there are inadequate data to support the superiority of any of the three urinary diversions in regard to HRQoL. ${ }^{73,78}$ There are advantages and disadvantages of each procedure; thus, it is of the utmost importance to provide patients with adequate information about each surgical approach to allow individuals to make an informed decision. ${ }^{78}$ Decreased body image, increased self-consciousness, and decreases in travel and activity levels are frequently experienced by patients with ileal conduits, whereas those with continent diversions report urinary symptoms related to use of a catheter. ${ }^{68,78}$ Among patients who underwent construction of orthotropic neobladders, night-time leakage can negatively impact HRQoL. ${ }^{68}$ In addition, acute and long-term adverse effects related to systemic chemotherapy and radiotherapy for treatment of MIBC may also affect HRQoL. ${ }^{24,44}$

\section{"Staging the aging" to assist with decision-making for management of bladder cancer}

The older patient population with $\mathrm{BC}$ poses a challenge in decision-making about various treatment interventions. ${ }^{79}$ 
Older patients, especially those with a smoking history, are much more likely to have underlying health issues that can increase complications and reduce the efficacy of cancer interventions. The decision to treat $\mathrm{BC}$ should be based on high-quality data from studies that identify which subset of older patients would most likely derive improved clinical outcomes. In other words, "fit" older patients, no matter what age, should be considered for aggressive interventions for $\mathrm{BC}$, while those with significant health issues may not benefit. In general, clinical trial data that establish the safety and efficacy of cancer treatment (surgery and chemotherapy) include patients who are on average 10 years younger than those with the disease in the general community and include patients without significant health status issues (ie, minimal comorbidity and good performance status). Therefore, little is known regarding the safety and efficacy of standard treatment regimens in older patients, especially those who are aged $\geq 75$ years and have other health status issues. ${ }^{36}$

A decision framework addressing BC treatment in older patients should balance underlying health considerations and risk of therapies with aggressiveness of the cancer. It is important to evaluate whether the patient is likely to derive survival or symptomatic benefit from management of BC. For example, the benefits of treatment may outweigh the risks for an older patient with aggressive or systemic disease, even if that patient has multiple other medical conditions impacting life expectancy. On the other hand, an older patient with a short life expectancy may not benefit from treatment and may be more vulnerable to harms from treatment, due to underlying health issues. Medical comorbidities may increase the risk of adverse outcomes (such as mortality from surgery or chemotherapy toxicity) that may decrease life expectancy rather than lengthen it. Patient comorbidities, functional impairments or mobility disability, and geriatric syndromes (including cognitive impairment) provide clinically useful, reasonably accurate estimates of life expectancy as well as a comprehensive evaluation of health status. ${ }^{80}$ The prevalence of comorbidity is known to be higher in older patients with $\mathrm{BC}$ and impacts the receipt and outcomes of cystectomy and perioperative chemotherapy. ${ }^{81}$ Unfortunately, the independent effects of other health status considerations such as functional impairments, mobility disability, and geriatric syndromes have not been well studied in older patients with BC. These factors, however, have been independently associated with a higher risk of surgical complications and morbidity and mortality from chemotherapy in other cancer populations. ${ }^{82,83}$ For example, sarcopenia, as measured by decreased lumbar skeletal muscle thickness, was independently associated with all-cause mortality (hazard ratio $1.93 ; P=0.004$ ) on multivariable analysis in over 200 patients who were to undergo cystectomy. ${ }^{84}$

Commonly used GA tools, which include both patientreported outcomes and objective tests of functional status, may assist with decision-making for the older adult with $\mathrm{BC}$. The GA is a multicomponent assessment of an older individual, which includes measurements of functional status, cognitive status, comorbidities, self-assessed health status, mobility, nutritional status, psychological status, and social circumstances (Table 3). ${ }^{82,85}$ The National Comprehensive Cancer Network Guidelines recommend all cancer patients aged $\geq 70$ years undergo some form of GA ${ }^{86} \mathrm{GA}$ has been validated for use in oncology clinical practice and trials. ${ }^{87,88}$ GA can help identify previously unrecognized conditions that can affect treatment tolerance and efficacy, including surgical outcomes. Identifying areas of vulnerability may assist in decision-making for the older cancer patient facing surgical intervention. For example, studies of older cancer patients undergoing elective oncologic surgery reported that worse geriatric depression score, cognitive impairment, and decreased functional status (OR 4.02, 95\% CI 1.24-13.09) independently predicted worse 30-day and 90-day major complication rates, and increased likelihood of discharge to a facility (OR 3.16, 95\% CI 1.99-13.02). ${ }^{89-91}$ Frailty was also associated with longer postoperative hospitalization and greater mortality at 30 and 90 days (OR 4.0, 95\% CI 1.1-15.2 and OR 3.0, 95\% CI 1.3-7.4, respectively). ${ }^{91}$

It is known that GA can identify those patients at highest risk for serious toxicities from chemotherapy. ${ }^{35}$ For example, the Cancer and Aging Research Group evaluated 500 patients prospectively with a comprehensive GA and identified eleven factors that were independently associated with chemotherapy toxicity, including genitourinary malignancies (Table 2).$^{35}$ A risk stratification tool was developed that was predictive of the incidence of chemotherapy toxicity and found this model to be superior to the Karnofsky Performance Status, the existing standard use by oncologists for assessment of fitness. In addition to predicting the risk for chemotherapy toxicity, GA can help identify patients with cognitive impairment, which has implications for consent to any form of treatment and increases risk from therapies such as surgery and chemotherapy. ${ }^{92}$

In order to help stratify older patients with $\mathrm{BC}$ for interventions, information from the GA can categorize patients into three groups that correlate with life expectancy. ${ }^{93}$ Patients who are "fit" have no significant functional 
Table 3 Geriatric assessment and measurement options

\begin{tabular}{|c|c|c|}
\hline Domains & Definition & Measurement options \\
\hline Function/physical & Ability to take care of one's own needs to live & Activities of daily living \\
\hline \multirow[t]{5}{*}{ performance } & independently at home & Instrumental activities of daily living \\
\hline & Ability to care for tasks that allow independence in & History of falls \\
\hline & the community & Timed Up and Go test \\
\hline & Physical performance is an objective evaluation of & Short Physical Performance Battery \\
\hline & mobility, balance, and fall risk & Handgrip testing \\
\hline \multirow[t]{5}{*}{ Comorbidity/pharmacy } & Chronic diseases that influence life expectancy and & Charlson Comorbidity Index \\
\hline & may influence tolerance to cancer treatment & Cumulative IIIness Scale-Geriatrics \\
\hline & Medications can increase risk of adverse events with & Comorbidity count and severity \\
\hline & cancer treatment & Medication count \\
\hline & & Beers criteria \\
\hline \multirow[t]{4}{*}{ Cognition } & Common in older patients and may affect decision- & Mini-Mental State Examination \\
\hline & making capacity and interfere with cancer treatment & Blessed-Orientation-Memory Scale \\
\hline & & Short Portable Mental Status Questionnaire \\
\hline & & Montreal Cognitive Assessment \\
\hline \multirow[t]{2}{*}{ Psychological status } & Depression and anxiety are independently associated & Geriatric Depression Scale \\
\hline & with adverse outcomes in cancer patients & Hospital Anxiety and Depression Scale \\
\hline \multirow[t]{3}{*}{ Nutrition } & Weight loss and anorexia affect tolerance to & Mini-Nutritional Assessment \\
\hline & treatment and survival in older cancer patients & Weight loss \\
\hline & & Body mass index \\
\hline \multirow[t]{3}{*}{ Social support } & Adequate social support necessary for older patients & Needs assessment of financial capabilities, \\
\hline & to successfully undergo treatment & transportation, and caregiver status \\
\hline & & Medical Outcomes Survey Social Support \\
\hline
\end{tabular}

impairments and/or comorbidities. Fit patients should receive evidence-based care as much as possible. On the other end of the spectrum, older patients who are "frail" demonstrate dependence in basic functional tasks, significantly impaired mobility, significant comorbidities, and/or at least one significant geriatric syndrome, such as falls. ${ }^{89}$ These patients are at high risk for toxicities from cancer treatment. Decisionmaking is more complex for patients who are vulnerable and have concomitant mild functional or cognitive issues, wellcontrolled and non-life threatening comorbid conditions, and/ or depression. Targeted interventions can be implemented with the goal of improving outcomes from cancer-directed therapy. As an example, "prehabilitation" to improve physical functioning may improve the likelihood of tolerating treatment for patients with mobility disorders. ${ }^{90}$ Although what is presented here needs to be validated in prospective studies, the framework described above can inform clinical decision-making in the elderly BC population where data are limited.

\section{Conclusion}

Active areas of investigation in the treatment of elderly patients with $\mathrm{BC}$ include the development of tools to characterize functional status beyond chronologic age. Using these tools in risk stratification of this heterogeneous population informs medical decision-making and reduces treatmentrelated complications. Identification of novel systemic therapies with increased efficacy against BC, development of surgical and radiation techniques that minimize morbidity, and clinical trials designed to ameliorate the impact of $\mathrm{BC}$ and $\mathrm{BC}$-directed treatment on HRQoL promise to improve the lives of all patients living with $\mathrm{BC}$.

\section{Disclosure}

The authors report no conflicts of interest in this work.

\section{References}

1. Surveillance Research Program. SEER Stat Fact Sheets: Bladder. National Cancer Institute; 2013. Available from: http://seer.cancer.gov/ statfacts/html/urinb.html. Accessed April 12, 2015.

2. International Bladder Cancer Nomogram Consortium, Bochner BH, Kattan MW, Vora KC. Postoperative nomogram predicting risk of recurrence after radical cystectomy for bladder cancer. J Clin Oncol. 2006;24(24):3967-3972.

3. Loehrer PJ Sr, Einhorn LH, Elson PJ, et al. A randomized comparison of cisplatin alone or in combination with methotrexate, vinblastine, and doxorubicin in patients with metastatic urothelial carcinoma: a cooperative group study. J Clin Oncol. 1992;10(7):1066-1073.

4. Siegel R, Naishadham D, Jemal A. Cancer statistics, 2013. CA Cancer J Clin. 2013;63(1):11-30.

5. Novara G, Catto JW, Wilson T, et al. Systematic review and cumulative analysis of perioperative outcomes and complications after robot-assisted radical cystectomy. Eur Urol. 2015;67(3):376-401.

6. Prout GR Jr, Wesley MN, Yancik R, Ries LA, Havlik RJ, Edwards BK. Age and comorbidity impact surgical therapy in older bladder carcinoma patients: a population-based study. Cancer. 2005;104(8):1638-1647.

7. Schiffmann J, Gandaglia G, Larcher A, et al. Contemporary 90-day mortality rates after radical cystectomy in the elderly. Eur J Surg Oncol. 2014;40(12):1738-1745. 
8. Fairey A, Chetner M, Metcalfe J, et al. Associations among age, comorbidity and clinical outcomes after radical cystectomy: results from the Alberta Urology Institute Radical Cystectomy Database. J Urol. 2008;180(1):128-134.

9. Nielsen ME, Shariat SF, Karakiewicz PI, et al. Advanced age is associated with poorer bladder cancer-specific survival in patients treated with radical cystectomy. Eur Urol. 2007;51(3):699-706.

10. Froehner M, Brausi MA, Herr HW, Muto G, Studer UE. Complications following radical cystectomy for bladder cancer in the elderly. Eur Urol. 2009;56(3):443-454.

11. Gilbert SM, Lai J, Saigal CS, Gore JL; Urologic Diseases in America Project. Downstream complications following urinary diversion. J Urol. 2013;190(3):916-922.

12. Sogni F, Brausi M, Frea B, et al. Morbidity and quality of life in elderly patients receiving ileal conduit or orthotopic neobladder after radical cystectomy for invasive bladder cancer. Urology. 2008;71(5): 919-923.

13. Clark PE, Stein JP, Groshen SG, et al. Radical cystectomy in the elderly: comparison of clinical outcomes between younger and older patients. Cancer. 2005;104(1):36-43.

14. Tang K, Li H, Xia D, et al. Laparoscopic versus open radical cystectomy in bladder cancer: a systematic review and meta-analysis of comparative studies. PLoS One. 2014;9(5):e95667.

15. Lin T, Fan X, Zhang C, et al. A prospective randomised controlled trial of laparoscopic vs open radical cystectomy for bladder cancer: perioperative and oncologic outcomes with 5-year follow-up. $\mathrm{Br} J$ Cancer. 2014;110(4):842-849.

16. Nix J, Smith A, Kurpad R, Nielsen ME, Wallen EM, Pruthi RS. Prospective randomized controlled trial of robotic versus open radical cystectomy for bladder cancer: perioperative and pathologic results. Eur Urol. 2010;57(2):196-201.

17. Niegisch G, Albers P, Rabenalt R. Perioperative complications and oncological safety of robot-assisted (RARC) vs open radical cystectomy (ORC). Urol Oncol. 2014;32(7):966-974.

18. Nguyen DP, Awamlh BA, Charles Osterberg E, et al. Postoperative complications and short-term oncological outcomes of patients aged $\geq 80$ years undergoing robot-assisted radical cystectomy. World $J$ Urol. November 20, 2014. [Epub ahead of print].

19. Clayman RH, Shipley WU, Galland-Girodet S, et al. Outcomes of selective bladder preservation in the elderly treated with conservative surgery and chemoradiation. Int J Radiat Oncol Biol Phys. 2013; 87(2):S83.

20. Gray PJ, Fedewa SA, Shipley WU, et al. Use of potentially curative therapies for muscle-invasive bladder cancer in the united states: results from the National Cancer Database. Eur Urol. 2013;63(5):823-829.

21. Chen RC, Shipley WU, Efstathiou JA, Zietman AL. Trimodality bladder preservation therapy for muscle-invasive bladder cancer. J Natl Compr Canc Netw. 2013;11(8):952-960.

22. James ND, Hussain SA, Hall E, et al. Radiotherapy with or without chemotherapy in muscle-invasive bladder cancer. $N$ Engl $\mathrm{J} \mathrm{Med.}$ 2012;366(16):1477-1488.

23. Mak RH, Hunt D, Shipley WU, et al. Long-term outcomes in patients with muscle-invasive bladder cancer after selective bladder-preserving combined-modality therapy: a pooled analysis of Radiation Therapy Oncology Group protocols 8802, 8903, 9506, 9706, 9906, and 0233. J Clin Oncol. 2014;32(34):3801-3809.

24. Efstathiou JA, Bae K, Shipley WU, et al. Late pelvic toxicity after bladder-sparing therapy in patients with invasive bladder cancer: RTOG 89-03, 95-06, 97-06, 99-06. J Clin Oncol. 2009;27(25):4055-4061.

25. Zietman AL, Sacco D, Skowronski U, et al. Organ conservation in invasive bladder cancer by transurethral resection, chemotherapy and radiation: results of a urodynamic and quality of life study on long-term survivors. J Urol. 2003;170(5):1772-1776.

26. Duchesne GM, Bolger JJ, Griffiths GO, et al. A randomized trial of hypofractionated schedules of palliative radiotherapy in the management of bladder carcinoma: results of Medical Research Council trial BA09. Int J Radiat Oncol Biol Phys. 2000;47(2):379-388.
27. Kouloulias V, Tolia M, Kolliarakis N, Siatelis A, Kelekis N. Evaluation of acute toxicity and symptoms palliation in a hypofractionated weekly schedule of external radiotherapy for elderly patients with muscular invasive bladder cancer. Int Braz J Urol. 2013;39(1): 77-82.

28. Advanced Bladder Cancer (ABC) Meta-analysis Collaboration. Neoadjuvant chemotherapy in invasive bladder cancer: update of a systematic review and meta-analysis of individual patient data advanced bladder cancer (ABC) meta-analysis collaboration. Eur Urol. 2005;48(2):202-205.

29. Grossman HB, Natale RB, Tangen CM, et al. Neoadjuvant chemotherapy plus cystectomy compared with cystectomy alone for locally advanced bladder cancer. $N$ Engl J Med. 2003;349(9): 859-866.

30. Sternberg CN, Skoneczna IA, Kerst JM, et al. Final results of EORTC Intergroup randomized phase III trial comparing immediate versus deferred chemotherapy after radical cystectomy in patients with pT3T4 and/or N+ M0 transitional cell carcinoma (TCC) of the bladder. J Clin Oncol. 2014;32(5 Suppl):Abstr 4500.

31. Clark PE, Agarwal N, Biagioli MC, et al. Bladder cancer (version 2.2014). Available from: www.nccn.org. Accessed April 11, 2015.

32. Raj GV, Karavadia S, Schlomer B, et al. Contemporary use of perioperative cisplatin-based chemotherapy in patients with muscle-invasive bladder cancer. Cancer. 2011;117(2):276-282.

33. Rehman S, Crane A, Din R, et al. Understanding avoidance, refusal, and abandonment of chemotherapy before and after cystectomy for bladder cancer. Urology. 2013;82(6):1370-1375.

34. Montgomery JS, Miller DC, Weizer AZ. Quality indicators in the management of bladder cancer. J Natl Compr Canc Netw. 2013;11(4): 492-500.

35. Hurria A, Togawa K, Mohile SG, et al. Predicting chemotherapy toxicity in older adults with cancer: a prospective multicenter study. J Clin Oncol. 2011;29(25):3457-3465.

36. Hutchins LF, Unger JM, Crowley JJ, Coltman CA Jr, Albain KS. Underrepresentation of patients 65 years of age or older in cancer-treatment trials. N Engl J Med. 1999;341(27):2061-2067.

37. Galsky MD. How I treat bladder cancer in elderly patients. $J$ Geriatr Oncol. 2015;6(1):1-7.

38. Sonpavde G, Watson D, Tourtellott M, et al. Administration of cisplatin-based chemotherapy for advanced urothelial carcinoma in the community. Clin Genitourin Cancer. 2012;10(1):1-5.

39. Galsky MD, Chen GJ, Oh WK, et al. Comparative effectiveness of cisplatin-based and carboplatin-based chemotherapy for treatment of advanced urothelial carcinoma. Ann Oncol. 2012;23(2):406-410.

40. Galsky MD, Hahn NM, Rosenberg J, et al. Treatment of patients with metastatic urothelial cancer "unfit" for cisplatin-based chemotherapy. J Clin Oncol. 2011;29(17):2432-2438.

41. Galsky MD, Hahn NM, Rosenberg J, et al. A consensus definition of patients with metastatic urothelial carcinoma who are unfit for cisplatinbased chemotherapy. Lancet Oncol. 2011;12(3):211-214.

42. Chau C, Wheater M, Geldart T, Crabb SJ. Clinical outcomes following neoadjuvant cisplatin-based chemotherapy for bladder cancer in elderly compared with younger patients. Eur J Cancer Care (Engl). 2015;24(2):155-162.

43. Galsky MD, Krege S, Lin CC, et al. Cisplatin-based combination chemotherapy in septuagenarians with metastatic urothelial cancer. Urol Oncol. 2014;32(1):30.e15-30.e21.

44. von der Maase H, Hansen SW, Roberts JT, et al. Gemcitabine and cisplatin versus methotrexate, vinblastine, doxorubicin, and cisplatin in advanced or metastatic bladder cancer: results of a large, randomized, multinational, multicenter, phase III study. J Clin Oncol. 2000;18(17):3068-3077.

45. De Santis M, Bellmunt J, Mead G, et al. Randomized phase II/III trial assessing gemcitabine/carboplatin and methotrexate/carboplatin/ vinblastine in patients with advanced urothelial cancer who are unfit for cisplatin-based chemotherapy: EORTC study 30986. J Clin Oncol. 2012;30(2):191-199. 
46. Sonpavde G, Galsky MD, Latini D, Chen GJ. Cisplatin-ineligible and chemotherapy-ineligible patients should be the focus of new drug development in patients with advanced bladder cancer. Clin Genitourin Cancer. 2014;12(2):71-73.

47. McCaffrey JA, Hilton S, Mazumdar M, et al. Phase II trial of docetaxel in patients with advanced or metastatic transitional-cell carcinoma. J Clin Oncol. 1997;15(5):1853-1857.

48. Vaughn DJ, Broome CM, Hussain M, Gutheil JC, Markowitz AB. Phase II trial of weekly paclitaxel in patients with previously treated advanced urothelial cancer. J Clin Oncol. 2002;20(4):937-940.

49. Sweeney CJ, Roth BJ, Kabbinavar FF, et al. Phase II study of pemetrexed for second-line treatment of transitional cell cancer of the urothelium. J Clin Oncol. 2006;24(21):3451-3457.

50. Witte RS, Elson P, Bono B, et al. Eastern Cooperative Oncology Group Phase II trial of ifosfamide in the treatment of previously treated advanced urothelial carcinoma. J Clin Oncol. 1997;15(2): 589-593.

51. Cancer Genome Atlas Research Network. Comprehensive molecular characterization of urothelial bladder carcinoma. Nature. 2014;507(7492):315-322.

52. Choi W, Porten S, Kim S, et al. Identification of distinct basal and luminal subtypes of muscle-invasive bladder cancer with different sensitivities to frontline chemotherapy. Cancer Cell. 2014;25(2): 152-165.

53. Damrauer JS, Hoadley KA, Chism DD, et al. Intrinsic subtypes of high-grade bladder cancer reflect the hallmarks of breast cancer biology. Proc Natl Acad Sci U S A. 2014;111(8):3110-3115.

54. Iyer G, Hanrahan AJ, Milowsky MI, et al. Genome sequencing identifies a basis for everolimus sensitivity. Science. 2012;338(6104):221.

55. Wagle N, Grabiner BC, Van Allen EM, et al. Activating mTOR mutations in a patient with an extraordinary response on a phase I trial of everolimus and pazopanib. Cancer Discov. 2014;4(5):546-553.

56. Iyer G, Al-Ahmadie H, Schultz N, et al. Prevalence and co-occurrence of actionable genomic alterations in high-grade bladder cancer. J Clin Oncol. 2013;31(25):3133-3140.

57. Ross JS, Wang K, Al-Rohil RN, et al. Advanced urothelial carcinoma: next-generation sequencing reveals diverse genomic alterations and targets of therapy. Mod Pathol. 2014;27(2):271-280.

58. Herbst RS, Soria JC, Kowanetz M, et al. Predictive correlates of response to the anti-PD-L1 antibody MPDL3280A in cancer patients. Nature. 2014;515(7528):563-567.

59. Powles T, Eder JP, Fine GD, et al. MPDL3280A (anti-PD-L1) treatment leads to clinical activity in metastatic bladder cancer. Nature. 2014;515(7528):558-562.

60. McDermott J, Jimeno A. Pembrolizumab: PD-1 inhibition as a therapeutic strategy in cancer. Drugs Today (Barc). 2015;51(1):7-20.

61. Robert C, Ribas A, Wolchok JD, et al. Anti-programmed-deathreceptor-1 treatment with pembrolizumab in ipilimumab-refractory advanced melanoma: a randomised dose-comparison cohort of a phase 1 trial. Lancet. 2014;384(9948):1109-1117.

62. Fung C, Pandya C, Guancial E, et al. Impact of bladder cancer on health related quality of life in 1,476 older Americans: a cross-sectional study. J Urol. 2014;192(3):690-695.

63. Messing EM, Madeb R, Feng C, et al. Grade and stage at presentation do not predict mortality in patients with bladder cancer who survive their disease. J Clin Oncol. 2009;27(15):2443-2449.

64. Noon AP, Albertsen PC, Thomas F, Rosario DJ, Catto JW. Competing mortality in patients diagnosed with bladder cancer: evidence of undertreatment in the elderly and female patients. $\mathrm{Br} J$ Cancer. 2013;108(7):1534-1540.

65. Karvinen KH, Courneya KS, North S, Venner P. Associations between exercise and quality of life in bladder cancer survivors: a population-based study. Cancer Epidemiol Biomarkers Prev. 2007;16(5):984-990.

66. Wildes TM, Ruwe AP, Fournier C, et al. Geriatric assessment is associated with completion of chemotherapy, toxicity, and survival in older adults with cancer. J Geriatr Oncol. 2013;4(3):227-234.
67. Apolo AB, Hoffman V, Kaag MG, et al. Summary of the 8th Annual Bladder Cancer Think Tank: collaborating to move research forward. Urol Oncol. 2015;33(2):53-64.

68. Botteman MF, Pashos CL, Hauser RS, Laskin BL, Redaelli A. Quality of life aspects of bladder cancer: a review of the literature. Qual Life Res. 2003;12(6):675-688.

69. Parkinson JP, Konety BR. Health related quality of life assessments for patients with bladder cancer. $J$ Urol. 2004;172(6 Pt 1): 2130-2136.

70. Aaronson NK, Ahmedzai S, Bergman B, et al. The European Organization for Research and Treatment of Cancer QLQ-C30: a quality-of-life instrument for use in international clinical trials in oncology. $J$ Natl Cancer Inst. 1993;85(5):365-376.

71. Cella DF, Tulsky DS, Gray G, et al. The Functional Assessment of Cancer Therapy scale: development and validation of the general measure. J Clin Oncol. 1993;11(3):570-579.

72. Singer S, Ziegler C, Schwalenberg T, Hinz A, Gotze H, Schulte T. Quality of life in patients with muscle invasive and non-muscle invasive bladder cancer. Support Care Cancer. 2013;21(5):1383-1393.

73. Mohamed NE, Diefenbach MA, Goltz HH, et al. Muscle invasive bladder cancer: from diagnosis to survivorship. Adv Urol. 2012;2012:142135.

74. Allareddy V, Kennedy J, West MM, Konety BR. Quality of life in longterm survivors of bladder cancer. Cancer. 2006;106(11):2355-2362.

75. Gilbert SM, Wood DP, Dunn RL, et al. Measuring health-related quality of life outcomes in bladder cancer patients using the Bladder Cancer Index (BCI). Cancer. 2007;109(9):1756-1762.

76. Reeve BB, Potosky AL, Smith AW, et al. Impact of cancer on health-related quality of life of older Americans. $J$ Natl Cancer Inst. 2009;101(12):860-868.

77. Norman GR, Sloan JA, Wyrwich KW. Interpretation of changes in health-related quality of life: the remarkable universality of half a standard deviation. Med Care. 2003;41(5):582-592.

78. Roaldsen M, Aarsaether E, Knutsen T, Patel HR. Strategies to improve quality of life in bladder cancer patients. Expert Rev Pharmacoecon Outcomes Res. 2014;14(4):537-544.

79. Dale W. "Staging the aging" when considering androgen deprivation therapy for older men with prostate cancer. J Clin Oncol. 2009;27(21):3420-3422.

80. Walter LC, Covinsky KE. Cancer screening in elderly patients: a framework for individualized decision making. JAMA. 2001; 285(21):2750-2756.

81. Goossens-Laan CA, Leliveld AM, Verhoeven RH, et al. Effects of age and comorbidity on treatment and survival of patients with muscleinvasive bladder cancer. Int J Cancer. 2014;135(4):905-912.

82. Mohile S, Dale W, Hurria A. Geriatric oncology research to improve clinical care. Nat Rev Clin Oncol. 2012;9(10):571-578.

83. Koroukian SM, Xu F, Bakaki PM, Diaz-Insua M, Towe TP, Owusu C. Comorbidities, functional limitations, and geriatric syndromes in relation to treatment and survival patterns among elders with colorectal cancer. J Gerontol A Biol Sci Med Sci. 2010;65(3): 322-329.

84. Psutka SP, Carrasco A, Schmit GD, et al. Sarcopenia in patients with bladder cancer undergoing radical cystectomy: impact on cancerspecific and all-cause mortality. Cancer. 2014;120(18):2910-2918.

85. Pal SK, Katheria V, Hurria A. Evaluating the older patient with cancer: understanding frailty and the geriatric assessment. CA Cancer J Clin. 2010;60(2):120-132.

86. Hurria A, Browner IS, Cohen HJ, et al. Senior adult oncology. J Natl Compr Canc Netw. 2012;10(2):162-209.

87. Hurria A, Gupta S, Zauderer M, et al. Developing a cancer-specific geriatric assessment: a feasibility study. Cancer. 2005;104(9):1998-2005.

88. Hurria A, Cohen HJ, Extermann M. Geriatric oncology research in the cooperative groups: a report of a SIOG special meeting. J Geriatr Oncol. 2010;1(1):40-44.

89. Balducci L, Extermann M. Management of the frail person with advanced cancer. Crit Rev Oncol Hematol. 2000;33(2):143-148. 
90. Gill TM, Baker DI, Gottschalk M, et al. A prehabilitation program for physically frail community-living older persons. Arch Phys Med Rehabil. 2003;84(3):394-404.

91. Hewitt J, Moug SJ, Middleton M, et al. Prevalence of frailty and its association with mortality in general surgery. Am J Surg. 2015;209(2): 254-259.
92. Ketelaars L, Pottel L, Lycke M, et al. Use of the Freund clock drawing test within the Mini-Cog as a screening tool for cognitive impairment in elderly patients with or without cancer. J Geriatr Oncol. 2013;4(2):174-182.

93. Mohile SG, Xian Y, Dale W, et al. Association of a cancer diagnosis with vulnerability and frailty in older Medicare beneficiaries. $J$ Natl Cancer Inst. 2009;101(17):1206-1215.

\section{Publish your work in this journal}

Clinical Interventions in Aging is an international, peer-reviewed journal focusing on evidence-based reports on the value or lack thereof of treatments intended to prevent or delay the onset of maladaptive correlates of aging in human beings. This journal is indexed on PubMed Central, MedLine,

\section{Dovepress}

CAS, Scopus and the Elsevier Bibliographic databases. The manuscript management system is completely online and includes a very quick and fair peer-review system, which is all easy to use. Visit http://www.dovepress. $\mathrm{com} /$ testimonials.php to read real quotes from published authors. 